\title{
MANOBRAS EVASIVAS ESTATÍSTICAS EM AMBIENTE DE DETRITOS ESPACIAIS SUJEITAS AO ARRASTO ATMOSFÉRICO
}

\section{Lucas dos Santos Ferreira ${ }^{1}$; Antônio Delson C. de Jesus²;}

1. Bolsista PIBIC-CNPq, Graduando em Física, Universidade Estadual de Feira de Santana, email: 1sfferreira@live.com

2. Orientador, Departamento de Física, Universidade Estadual de Feira de Santana, email: a1d1j1@gmail.com

PALAVRAS-CHAVE: Manobras Evasivas, Detritos Espaciais, Força Dissipativa.

\section{INTRODUÇÃO}

O estudo da dinâmica de missões espaciais em ambiente de crescimento e evolução de detritos espaciais (DE) tornou-se fundamental para se atingir objetivos importantes em todo mundo, desde meados do século XX. Devido a esse crescente número de detritos juntamente com o aumento das operações espaciais surge a necessidade de estudar as interações entre veículos e DE e buscar manobras evasivas, frente a colisões iminentes.

Neste trabalho, estudamos essas manobras em órbitas baixas da Terra (LEO) onde atua a força de arrasto atmosférico, que é uma força dissipativa. A expressão desta força é:

$$
\vec{f}_{\text {drag } D E}=-\frac{1}{2} \frac{C_{d} A_{D E}}{m_{D E}} \rho \vec{V}_{D E}\left|\vec{V}_{D E}\right|
$$

Esta força depende da velocidade do detrito em relação à atmosfera terrestre, $\mathrm{V}_{\mathrm{DE}}$, da densidade do ar, $\rho$, da taxa área por massa do detrito $(\mathrm{A} / \mathrm{m})$ e do coeficiente de arrasto, $\mathrm{C}_{\mathrm{d}}$. Podemos dizer que a força de arrasto é o resultado da interação entre os corpos e o movimento da atmosfera juntamente com a Terra e é também a principal força que atua em satélites artificiais em baixas altitudes terrestres.

\section{MATERIAL E MÉTODOS OU METODOLOGIA}

A metodologia utilizada em nossos estudos tratou-se da dedução das equações da dinâmica relativa entre os corpos envolvidos (DE e veículo espacial), considerando as forças gravitacional e o arrasto atmosférico. Logo após as deduções, realizamos as simulações numéricas das dinâmicas, com os objetivos de obtermos as condições iniciais angulares para posteriormente estudarmos as manobras evasivas.

\section{RESULTADOS E/OU DISCUSSÃO}

Os resultados obtidos permitiram determinar como as velocidades inicias relativas se distribuíram nos octantes da esfera celeste, tanto para o caso da dinâmica homogênea como para a sujeita a ação do arrasto atmosférico. Esses resultados podem ser observados nos gráficos das Figuras 1 e 2, a seguir. 

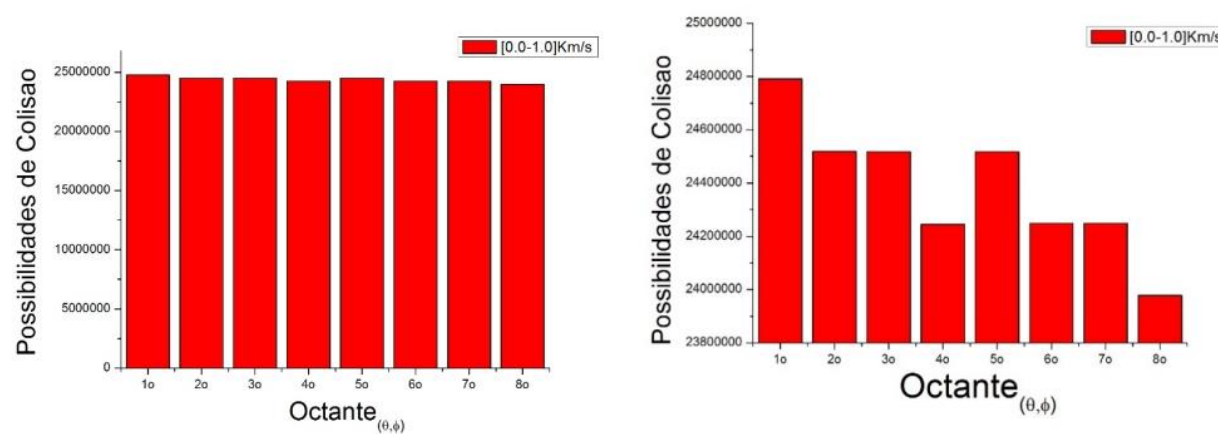

FIGURA (1)- Possibilidades de colisão vs. Octantes. Velocidades iniciais em $[0.0-1.0] \mathrm{Km} / \mathrm{s}$.

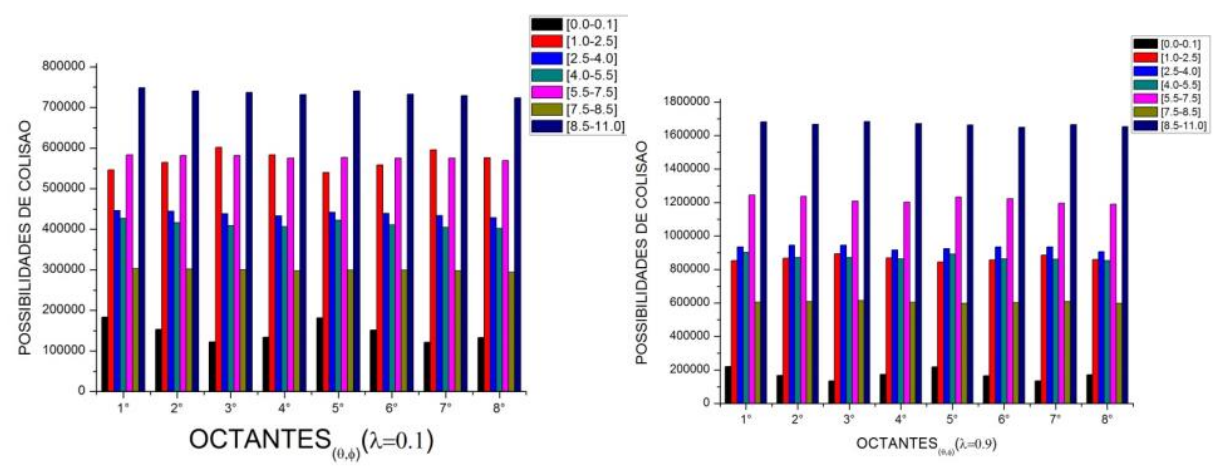

FIGURA (2)- Possibilidades de Colisão vs. Octantes vs. Velocidades Iniciais, $\lambda=0.1$ e $\lambda=0.9$, no intervalo de tempo de 1 a 1000 segundos, $\rho=2,789.10^{-10} \mathrm{Kg} / \mathrm{m}^{3}, r_{D E}=5.10^{-5} \mathrm{~km}$.

$$
m_{D E}=10^{-3} \mathrm{~kg}, C_{d}=2.2 \text {, }
$$

A solução da equação diferencial da dinâmica relativa, sujeita as forças de propulsão e de arrasto atmosférico foi encontrada. Suas componentes em Coordenadas Cartesianas são:

$$
\begin{aligned}
& X_{(t)}=e^{\frac{-\alpha_{2} t}{2}}\left\{\left\{\frac{x_{o}}{2}+\frac{\dot{x}_{o}}{2 k_{x}}-\frac{\left(\lambda^{2}\right)}{2 \alpha_{3}}+\frac{\alpha_{2}}{4 k_{x}}\left[x_{o}-\frac{\left(\lambda^{2}\right)}{\alpha_{3}}\right]\right\} e^{k_{x} t}\right. \\
& \left.+\left\{\frac{x_{o}}{2}-\frac{\dot{x}_{o}}{2 k_{x}}-\frac{\left(\lambda^{2}\right)}{2 \alpha_{3}}-\frac{\alpha_{2}}{4 k_{x}}\left[x_{o}-\frac{\left(\lambda^{2}\right)}{\alpha_{3}}\right]\right\} e^{-k_{x} t}\right\}+\frac{\left(\lambda^{2}\right)}{\alpha_{3}} \\
& Y_{(t)}=e^{\frac{-\alpha_{2} t}{2}}\left\{\left\{\frac{y_{o}}{2}\left[1+\frac{\alpha_{1}}{2 k_{y}}\right]+\frac{\left(\left(\lambda^{2}\right)+D\right)}{2 C}\left[1+\frac{\alpha_{1}}{2 k_{y}}\right]+\frac{\dot{y}_{o}}{2 k_{y}}\right\}^{k_{y} t}\right. \\
& \left.+\left\{\frac{y_{o}}{2}\left[1-\frac{\alpha_{1}}{2 k_{y}}\right]+\frac{\left(\left(\lambda^{2}\right)+D\right)}{2 C}\left[1-\frac{\alpha_{1}}{2 k_{y}}\right]-\frac{\dot{y}_{o}}{2 k_{y}}\right\}^{-k_{y} t}\right\}-\frac{\left(\left(\lambda^{2}\right)+D\right)}{C} \\
& Z_{(t)}=e^{\frac{-k t}{2}}\left\{z_{o} \cos \left(k_{z} t\right)+\left[\frac{\dot{z}_{o}}{k_{z}}+\frac{z_{o} K}{2 k_{2}}\right] \sin \left(k_{z} t\right)\right\}
\end{aligned}
$$

Além das condições que garantem a colisão com a dinâmica com arrasto e a gravidade:

$$
\begin{aligned}
\dot{X}_{o}=\frac{-2 k_{x}}{\left[e^{k_{x} t}-e^{-k_{x} t}\right]}\left\{\left\{\frac{x_{o}}{2}-\frac{\left(\lambda^{2}\right)}{2 \alpha_{3}}+\frac{\alpha_{2}}{4 k_{x}}\left[x_{o}-\frac{\left(\lambda^{2}\right)}{\alpha_{3}}\right]\right\} e^{k_{x} t}\right. \\
\left.+\left\{\frac{x_{o}}{2}-\frac{\left(\lambda^{2}\right)}{2 \alpha_{3}}-\frac{\alpha_{2}}{4 k_{x}}\left[x_{o}-\frac{\left(\lambda^{2}\right)}{\alpha_{3}}\right]\right\} e^{-k_{x} t}+\frac{\left(\lambda^{2}\right)}{\alpha_{3}} e^{\frac{\alpha_{x} t}{2}}\right\}
\end{aligned}
$$




$$
\begin{aligned}
& \dot{Y}_{o}=\frac{-2 k_{y}}{\left[e^{k} y^{t}-e^{-k} y^{t}\right]}\left\{\left\{\frac{y_{0}}{2}\left[1+\frac{\alpha_{1}}{2 k_{y}}\right]+\frac{\left(\left(\lambda^{2}\right)+D\right)}{2 C}\left[1+\frac{\alpha_{1}}{2 k_{y}}\right]\right\} e^{k_{y} t}+\left\{\frac{y_{0}}{2}\left[1-\frac{\alpha_{1}}{2 k_{y}}\right]+\frac{\left(\left(\lambda^{2}\right)+D\right)}{2 C}[1-\right.\right. \\
& \left.\left.\left.\frac{\alpha_{1}}{2 k_{y}}\right]\right\} e^{-k_{y} t}-\left(\frac{\left(\left(\lambda^{2}\right)+D\right)}{C} e^{\frac{\alpha_{1} t}{2}}\right)\right\} \\
& \dot{Z}_{o}=\frac{\omega}{\sin \left(k_{z} t\right)}\left\{-z_{o} \cos \left(k_{z} t\right)-\left[\frac{z_{o} k}{2 k_{2}}\right] \sin \left(k_{z} t\right)\right\}
\end{aligned}
$$

E por fim as equações que descrevem a atuação do sistema de propulsão sobre o satélite em ambiente de arrasto atmosférico:

$$
\begin{aligned}
& z_{(\mathrm{t})}=\left[z_{0} e^{\frac{-h t}{2}}\right] \cos \left(k_{z} t\right) \\
& +\left[\frac{\tilde{Z}_{0}}{k_{z}} e^{\frac{-h t}{2}}+\frac{z_{0} k}{2 k_{2}} e^{\frac{-h t}{2}}+\frac{v_{z}}{k_{2}} \ln \left(m_{0}\left(X+e^{-Y t}\right)\right)\left(1-e^{-\frac{k}{2} t}\right)-\frac{v_{z}}{k_{2}} e^{\frac{-h t}{2}} \ln \left(\frac{\chi+1}{\chi}\right)\right] \sin \left(k_{z} t\right) \\
& +v_{z} \sum_{n=1} \frac{k_{n n}}{\left(\left(\frac{A}{2}-\gamma n\right)^{2}+k_{2}^{2}\right)}\left[\left(\frac{k}{2}-\gamma n\right) e^{\frac{-A t}{2}}\left(\frac{k}{2 k_{2}} \operatorname{sen}\left(k_{2} t\right)-\cos \left(k_{2} t\right)\right)\right. \\
& \left.+k_{2}\left(\operatorname{sen}\left(k_{2} t\right)+\frac{k}{2 k_{2}} \cos \left(k_{2} t\right)\right)-\gamma n e^{\frac{-k t}{2}}\right] \\
& X_{(t)}=C_{1} e^{\left(k_{4}-\frac{\alpha_{2}}{2}\right) t}+C_{1} e^{-\left(k_{4}+\frac{\alpha_{2}}{2}\right) t}-\left[\frac{e^{\left(k_{4}-\frac{\alpha_{2}}{2}\right) t}-1}{\left(k_{4}-\frac{\alpha_{2}}{2}\right)}\right]\left[\frac{\lambda^{2}}{2 k_{4}}+\frac{v_{x}\left(k_{4}-\frac{\alpha_{2}}{2}\right) \ln \left(m_{o} \chi\right)}{2 k_{4}}\right] \\
& +\frac{v_{x}}{2 k_{4}}\left[e^{\left(k_{4}-\frac{\alpha_{2}}{2}\right) t} \ln \left(m_{\circ}(\chi+1)\right)-\ln \left(m_{o}\left(\chi+e^{-\gamma t}\right)\right)\right] \\
& -\frac{v_{x}\left(k_{4}-\frac{\alpha_{2}}{2}\right)}{2 k_{4}} \sum_{n=1}^{\infty}\left[\frac{e^{-\gamma n t}-e^{-\left(k_{4}-\frac{\alpha_{2}}{2}\right) t}}{\left(\left(\frac{\alpha_{1}}{2}\right)-k_{4}-(\gamma n)\right)}\right] \\
& Y_{(t)}=C_{3} e^{\left(k_{\mathrm{s}}-\frac{\alpha_{1}}{2}\right) t}+C_{4} e^{-\left(k_{\mathrm{B}}+\frac{\alpha_{1}}{2}\right) t}-\left[\frac{e^{\left(k_{\mathrm{B}}-\frac{\alpha_{1}}{2}\right) t}-1}{\left(k_{3}-\frac{\alpha_{1}}{2}\right)}\right]\left[\frac{\lambda^{2}+D}{2 k_{3}}+\frac{v_{y}\left(k_{3}-\frac{\alpha_{1}}{2}\right) \ln \left(m_{o} \chi\right)}{2 k_{3}}\right] \\
& +\frac{v_{y}}{2 k_{3}}\left[e^{\left(k_{\mathrm{a}}-\frac{\alpha_{1}}{2}\right) t} \ln \left(m_{o}(\chi+1)\right)-\ln \left(m_{o}\left(\chi+e^{-\gamma t}\right)\right)\right] \\
& -\frac{v_{y}\left(k_{3}-\frac{\alpha_{1}}{2}\right)}{2 k_{3}} \sum_{n=1}^{\infty}\left[\frac{e^{-\gamma n t}-e^{-\left(k_{3}-\frac{\alpha_{1}}{2}\right) t}}{\left(\left(\frac{\alpha_{1}}{2}\right)-k_{3}-(\gamma n)\right)}\right]
\end{aligned}
$$

Os gráficos da Figura 3 nos mostram o comportamento da dinâmica para o caso de colisão e manobra evasiva:
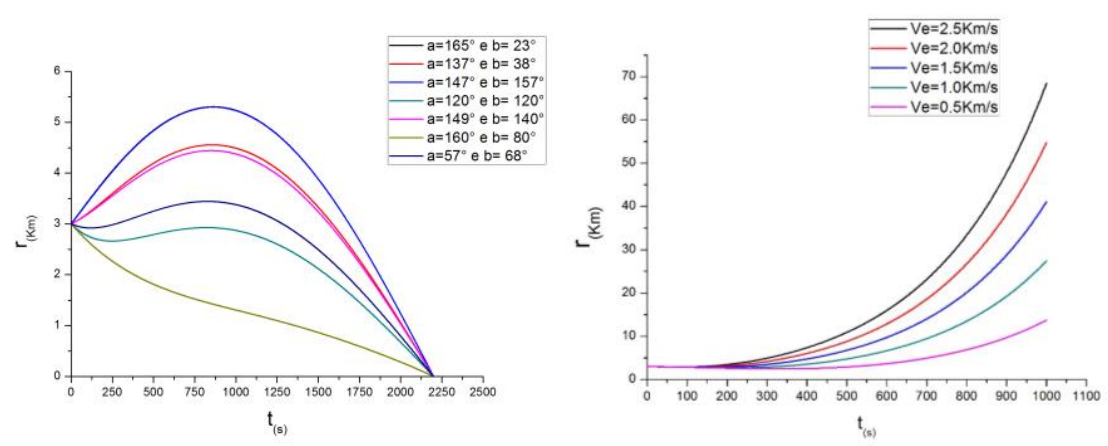

FIGURA (3)- (a) Dinâmica de colisão, para tc=2200s e (b) Dinâmica sujeita a ação da propulsão, para $t c=1000 \mathrm{~s}, \chi=10, \gamma=10^{-6}, \alpha=165^{\circ}$ e $\beta=23^{\circ}$. 


\section{CONSIDERAÇÕES FINAIS}

Diante de tudo que foi exposto, além das deduções das dinâmicas, foi possível concluir que as velocidades não estão distribuídas nos octantes de maneira homogêneo, pois existem regiões em que determinadas faixas de velocidades se apresentam com maior incidência. Sobre o arrasto e as condições inicias foi possível verificar a redistribuição das mesmas sobre o efeito do arrasto. Há maior possibilidade de colisão para velocidades maiores, sob ação desta força. Verificou-se também que naturalmente as condições com o arrasto não tem um caráter colisional e que valores de velocidade de exaustão baixas podem ser usados em ambiente com arrasto, pois a própria ação natural já atua acelerando os corpos e evitando assim as possíveis colisões.

\section{REFERÊNCIAS}

Cordelli, A., Farinella, P. and Rossi, A. The influence of the fragmentation threshold on the long term evolution of the orbital debris environment (submitted to Elsevier Preprint, 1997).

Davis, D.R., Farinella, P., Paolicchi, P., Weidenschilling, S.J, and Binzel, R.P.: 1989, Asteroid collisional history: Effects on sizes and spins, in Asteroids II, eds. R.P.Binzel, T.Gehrels and M.S. Matthews(Univ. of Arizona Press), pp.805-826.

Davis, D. R., Farinella, P., Paolicchi, P., Weidenschiling, S. J. and Binzel, R. P. Asteroid collisional history: Effects on sizes and spins, in Asteroids II, edited by R. P. Binzel, T. Gérels, and M. S. Mattews, 805-826, University of Arizona Press, Tucson, 1989.

Davis, D. R., Ryan, E. V. and Farinella, P. Asteroid collisional evolution: Results from current scaling algoritms, Planet Space Sci., 42, 599-610, 1994.

Kessler, D. J., Cour-Palais, B.G. Collision frequency of artificial satellite: The creation of a debris belt, J. Geophys. Res., 83, 2637-2646, 1978.

Kessler, D. J., Landry, P.M., Cour-Palais, B.G., Taylor, R.E. Collision Avoidance in Space, IEEE Spectrum, June 80, 1980.

Kessler, D. J., Collision cascading: Limits of population growth in low Earth orbit. Adv. Space Res., 11, 12, 63-66, 1991.

Rossi, A., Cordelli, A., Farinella, P. and Anselmo, L. Collisional evolution of the Earth's orbital debris cloud, J. Geophys. Res., 99, 23, 23.195-23.210, 1994.

Rossi, A., Cordelli, A., Farinella, P., Anselmo, L. and Pardini, C. Long term evolution of the space debris population, Adv. Space Res., 19, 2, 331-340, 1997. 